\begin{tabular}{|c|l|}
\hline Title & Operator-theoretical analysis of a representation of a supersymmetry al gebra in Hilbert space \\
\hline Author(s) & A rai, A sao \\
\hline Citation & Journal of Mathematical Physics, 36(2), 613-621 \\
\hline https://doi.org/10.1063/1.531144 \\
\hline Issue Date & 1995-02 \\
\hline Doc URL & http://hdl.handle.net/2115/13671 \\
\hline Rights & Copyright $\odot$ 1995 A merican Institute of Physics \\
\hline Type & article \\
\hline File Information & jmp36-2.pdf \\
\hline
\end{tabular}

Instructions for use 


\title{
Operator-theoretical analysis of a representation of a supersymmetry algebra in Hilbert space
}

\author{
Asao Arai \\ Department of Mathematics, Hokkaido University, Sapporo 060, Japan
}

(Received 11 July 1994; accepted for publication 9 September 1994)

\begin{abstract}
Operator-theoretical analysis is made on (unbounded) representations, in Hilbert spaces, of a supersymmetry (SUSY) algebra coming from a supersymmetric quantum field theory in two-dimensional space-time. A basic idea for the analysis is to apply the theory of strongly anticommuting self-adjoint operators. A theorem on integrability of a representation of the SUSY algebra is established. Moreover, it is shown that strong anticommutativity of self-adjoint operators is a natural and suitable concept in analyzing representations of the SUSY algebra in Hilbert space. (C) 1995 American Institute of Physics.
\end{abstract}

\section{INTRODUCTION}

In a relativistic supersymmetric quantum field theory (SSQFT) in two-dimensional spacetime, the generators $\left\{Q_{1}, Q_{2}\right\}$ of supersymmetry (SUSY), called supercharges, play an important role, forming, together with the generators $\{H, P\}$ of the space-time translation, the SUSY algebra $^{1}$

$$
Q_{1}^{2}=H+P, \quad Q_{2}^{2}=H-P, \quad Q_{1} Q_{2}+Q_{2} Q_{1}=0 .
$$

In this article we consider representations, in Hilbert spaces, of the SUSY algebra (1.1), concentrating our attention on their operator-theoretical contents. Let $\mathscr{H}$ be a Hilbert space and $\mathscr{D}$ be a dense subspace in $\mathscr{C}$. We say that the set $\left\{\mathscr{B}, \mathscr{D}, H, P, Q_{1}, Q_{2}\right\}$ is a symmetric representation of Eq. (1.1) if $H, P, Q_{1}$, and $Q_{2}$ are symmetric operators in $\mathscr{H}$, leave $\mathscr{D}$ invariant, and satisfy Eq. (1.1) on $\mathscr{Z}$. An interesting problem is then to formulate integrability conditions for a symmetric representation $\left\{\mathscr{H}, \mathscr{Q}, H, P, Q_{1}, Q_{2}\right\}$ of Eq. (1.1) in the sense that (i) $H, P, Q_{1}$, and $Q_{2}$ are essentially self-adjoint on $\mathscr{D}$ (denote their closures by $\bar{H}, \bar{P}, \bar{Q}_{1}$, and $\bar{Q}_{2}$, respectively); (ii) $\left\{\bar{H}, \bar{P}, \bar{Q}_{1}\right\}$ and $\left\{\bar{H}, \bar{P}, \bar{Q}_{2}\right\}$ are families of strongly commuting self-adjoint operators, respectively (see Definition 2.1(i) below); (iii) the pair $\{\bar{H}, \bar{P}\}$ satisfies

$$
\pm \bar{P} \leqslant \bar{H} \text {. }
$$

In the context of relativistic quantum field theory, where $\bar{H}$ and $\vec{P}$ denote the Hamiltonian and the momentum operator, respectively, the last condition together with the strong commutativity of $\bar{H}$ and $\bar{P}$ is called the spectral condition. Formulating such an integrability condition in an abstract form may also be useful in applications, in particular, in mathematically rigorous constructions of models of SSQFT. ${ }^{2-5}$

A key to analyze symmetric representations of the SUSY algebra is to note that the supercharges $Q_{1}$ and $Q_{2}$ anticommute in a "naive" sense [see the last relation in (1.1)]. In operator theory, there is a proper concept of anticommutativity for (unbounded) self-adjoint operators, which was introduced by Vasilescu ${ }^{6}$ and has been developed by some authors. ${ }^{7-11}$ In this article we call it strong anticommutativity for clarity [see Definition 2.1(ii) below]. Indeed, for unbounded self-adjoint operators, there is essential difference between strong anticommutativity and the ordinary "naive" anticommutativity [see Remark (2) after Definition 2.1]. ${ }^{12}$ Thus we are naturally led to apply the theory of strongly anticommuting self-adjoint operators to operatortheoretical analysis of symmetric representations of the SUSY algebra. This is the basic idea of our 
method and this viewpoint is new. ${ }^{13}$ We shall show that the concept of strong anticommutativity of self-adjoint operators is actually a natural concept to analyze representations of the SUSY algebra.

As is well known in the physics literature, ${ }^{14}$ the SUSY algebra (1.1) may be regarded as the Lie superalgebra of a "supergroup." In this connection, it may be desirable to formulate integrability conditions for representations of the SUSY algebra in such a way that $H, P, Q_{1}$, and $Q_{2}$ become the generators of a representation of the supergroup. However, the notion of a supergroup representation cannot be realized within Hilbert space formalism (one would need a concept like a "Hilbert module over an infinite-dimensional Grassmann algebra"). In this article, we do not discuss this aspect; we stay within the Hilbert space formalism and concentrate our attention on opcrator-theoretical contents of representations of the SUSY algebra in Hilbert spaces.

This article is organized as follows. In Sec. II we prove a theorem on integrability of a representation of the SUSY algebra (1.1). In Sec. III, we show that, under a natural condition, strong anticommutativity for the supercharges is necessary for a symmetric representation of Eq. (1.1) in which $\{H, P\}$ has a strongly commuting self-adjoint extension. We also give an example of nonintegrable symmetric representations of Eq. (1.1) in which the supercharges do not strongly anticommute. In the last section, we present a method to construct an integrable symmetric representation of Eq. (1.1) from two strongly anticommuting self-adjoint supercharges.

\section{INTEGRABILITY}

We first give a definition of strong (anti)commutativity of two self-adjoint operators.

Definition 2.1: Let $A$ and $B$ be self-adjoint operators in a Hilbert space.

(i) We say that $A$ and $B$ strongly commute if their spectral measures commute.

(ii) We say that $A$ and $B$ strongly anticommute if

$$
e^{i t A} B \subset B e^{-i t A}
$$

for all $t \in \mathbb{R}$.

Remark:

(1) Definition 2.1(ii) is symmetric in $A$ and $B$ (see Ref. 8).

(2) Let $A$ and $B$ be self-adjoint operators in a Hilbert space. If $A$ and $B$ are bounded, then the strong anticommutativity of $A$ and $B$ is equivalent to the ordinary anticommutativity of them: $A B+B A=0$. However, in the case where $A$ and $B$ are unbounded, these two concepts of anticommutativity are not equivalent; it can be shown that the concept of strong anticommutativity is certainly stronger than that of the ordinary anticommutativity. In fact, the following holds (see Ref. 6): If $A$ and $B$ strongly anticommute, then $D(A) \cap D(B) \cap D(A B)=D(A) \cap D(B) \cap D(B A)$ and

$$
(A B+B A) f=0, \quad f \in D(A) \cap D(B) \cap D(A B),
$$

where $D(T)$ denotes the domain of the operator $T$. However, the converse is not true. See Example 3.3 in Ref. 6 and the example in Sec. III below.

In what follows, the domain of the sum $A+B$ of two linear operators $A$ and $B$ in a Hilbert space is taken to be $D(A) \cap D(B)$, unless otherwise stated.

We shall prove the following:

Theorem 2.2: Let $\left\{\mathscr{H}, \mathscr{D}, H, P, Q_{1}, Q_{2}\right\}$ be a symmetric representation of Eq. (1.1). Suppose that $H$ is essentially self-adjoint on $\mathscr{Q}$ (its closure is denoted $\bar{H}$ ). Then the following (i)-(iii) hold:

(i) The symmetric operators $Q_{1} \pm Q_{2}, Q_{1}$ and $Q_{2}$ are essentially self-adjoint on $\mathscr{Z}$ and the closures $\bar{Q}_{1}$ and $\bar{Q}_{2}$ strongly anticommute. Moreover, the following operator equalities hold:

$$
\vec{H}=\frac{1}{2}\left(\overline{Q_{1} \pm Q_{2}}\right)^{2}=\frac{1}{2}\left(\bar{Q}_{1} \pm \bar{Q}_{2}\right)^{2}=\frac{1}{2}\left(\bar{Q}_{1}^{2}+\bar{Q}_{2}^{2}\right) .
$$


(ii) (Spectral condition) $P$ is essentially self-adjoint on $\mathscr{D}$ and $\bar{P}$ strongly commutes with $\bar{H}$. Moreover, $\{\bar{H}, \bar{P}\}$ satisfies Eq. (1.2).

(iii) Each $\bar{Q}_{j}(j=1,2)$ strongly commutes with $\bar{P}$ and $\bar{H}$.

Some remarks may be in order on this theorem:

(1) In constructing a model of SSQFT in a mathematically rigorous way, one usually starts with proving the essential self-adjointness of the Hamiltonian of the model on a suitable dense domain. ${ }^{15}$ Thus the assumption of Theorem 2.2 is natural from such a viewpoint. Moreover, Theorem 2.2 may be remarkable in the sense that it shows that we need only to assume the essential self-adjointness of $H$ on $\mathscr{L}$ to obtain all the desired properties for $\left\{H, P, Q_{1}, Q_{2}\right\}$ mentioned in the introduction as well as the strong anticommutativity of $\bar{Q}_{1}$ and $\bar{Q}_{2}$.

(2) Theorem 2.2(i) implies the following: if $Q_{1}$ and $Q_{2}$ are essentially self-adjoint on $\mathscr{D}$, but $\bar{Q}_{1}$ and $\bar{Q}_{2}$ do not strongly anticommute, then $H$ cannot be essentially self-adjoint on $\mathscr{D}$. This suggests that strong anticommutativity is a natural concept to characterize properties of the supercharges properly. In the next section, we shall discuss this aspect in some more detail.

(3) We can construct a symmetric representation $\left\{\mathscr{H}, \mathscr{D}, H, P, Q_{1}, Q_{2}\right\}$ of Eq. (1.1) in an abstract Boson-Fermion Fock space ${ }^{16}$ which satisfies the assumption of Theorem 2.2 and in which each $Q_{j}$ is an infinite-dimensional Dirac-type operator and $H$ and $P$ are second quantized operators. $^{17}$

To prove Theorem 2.2, we recall basic results in the theory of strongly anticommuting selfadjoint operators.

Lemma 2.3: (Refs. 6,8) Let $A$ and $B$ be strongly anticommuting self-adjoint operators in a Hilbert space. Then the following (i) and (ii) hold:

(i) The operators $A+B$ and $A^{2}+B^{2}$ are self-adjoint and the operator equality

$$
(A+B)^{2}=A^{2}+B^{2}
$$

holds.

(ii) $A$ (resp. B) and $|B|$ (resp. $|A|)$ strongly commute.

(iii) $|A|$ and $|B|$ strongly commute.

Theorem 2.4: Let $A$ and $B$ be symmetric operators in a Hilbert space $\mathscr{H}$ and let $\mathscr{O C}$ be $a$ dense subspace of $\mathscr{C}$ which is left invariant by $A$ and $B$. Assume that $A^{2}+B^{2}$ is essentially self-adjoint on $\mathscr{H}$ and $A B f+B A f=0$ for all $f \in \mathscr{H}$. Then $A \pm B, A$ and $B$ are essentially selfadjoint on. $\mathscr{H}$ and the closures of $A$ and $B$ strongly anticommute. Moreover, the following operator equalities hold:

$$
\overline{A^{2}+B^{2}}=(\overline{A \pm B})^{2} \text {. }
$$

Proof: Similar to the proof of Corollary 4.5 in Ref. 8.

Remark: The conclusion of Theorem 2.4 is slightly stronger than that of the corollary of the cited article in that $\mathscr{Z}$ is a common core for $A \pm B, A$ and $B$.

We also need the following lemma:

Lemma 2.5: (Ref. 18) Let $A$ he a self-adjoint operator and $B$ be a symmetric operator. Suppose that $D(A) \subset D(B)$ and $(A f, B g)=(B f, A g)$ for all $f, g \in D(A)$. Then $B$ is essentially self-adjoint and its closure strongly commutes with $A$.

Proof of Theorem 2.2:

(i) It follows from the first two equations in (1.1) that

$$
\begin{gathered}
H=\frac{1}{2}\left(Q_{1}^{2}+Q_{2}^{2}\right), \\
P=\frac{1}{2}\left(Q_{1}^{2}-Q_{2}^{2}\right)
\end{gathered}
$$


on $\mathscr{D}$. Hence, by the assumption, $Q_{1}^{2}+Q_{2}^{2}$ is essentially self-adjoint on $\mathscr{Z}$. Therefore we can apply Theorem 2.4 with $A=Q_{1}, B=Q_{2}$, and $\mathscr{A}=\mathscr{D}$ to obtain the first half of part (i) and the first equality in (2.1). It is easy to see that $\overline{Q_{1} \pm Q_{2}}=\bar{Q}_{1} \pm \bar{Q}_{2}$. Hence the first equality in (2.1) and Lemma 2.3 imply the second and third equalities in (2.1).

(ii) By the third equation in (1.1), we have

$$
\left(Q_{1}^{2} \phi, Q_{2}^{2} \phi\right)=\left(Q_{1} \phi, Q_{2}^{2} Q_{1} \phi\right) \geqslant 0, \quad \phi \in \mathscr{Q} .
$$

By this fact and Eq. (2.2), we obtain

$$
\left\|Q_{j}^{2} \phi\right\| \leqslant 2\|H \phi\|, \quad \phi \in \mathscr{D}, j=1,2 .
$$

By Eqs. (2.3) and (2.4), we have for all $\phi \in \mathscr{W}$

$$
\|P \phi\| \leqslant \frac{1}{2}\left(\left\|Q_{1}^{2} \phi\right\|+\left\|Q_{2}^{2} \phi\right\| \leqslant 2\|H \phi\| .\right.
$$

A direct computation shows that

$$
(P \phi, H \psi)=(H \phi, P \psi), \quad \phi, \psi \in \mathscr{Z} .
$$

By a limiting argument using Eq. (2.5) and the fact that $\mathscr{D}$ is a core for $\bar{H}$, we can show that $D(\bar{H}) \subset D(\vec{P})$ and

$$
(\bar{P} \phi, \vec{H} \psi)=(\bar{H} \phi, \bar{P} \psi), \quad \phi, \psi \in D(\tilde{H}),
$$

where $\bar{P}$ is the closure of $P$ restricted to $\mathscr{Z}$. Thus, by applying Lemma 2.5 with $A=\bar{H}$ and $B=\bar{P}$, we conclude that $\bar{P}$ is self-adjoint and strongly commutes with $\bar{H}$.

By Eqs. (2.2) and (2.3), we have

$$
(\psi, \pm \bar{P} \psi) \leqslant(\psi, \bar{H} \psi), \quad \psi \in \mathscr{D} .
$$

Then, by a limiting argument using Eq. (2.5), we can extend Eq. (2.6) to all $\psi \in D(\vec{H})$. Thus Eq. (1.2) follows.

(iii) By Lemma 2.3(ii), $\bar{Q}_{j}$ and $\bar{Q}_{k}^{2}$ strongly commute $(j, k=1,2)$. Hence, by the two variable functional calculus, we obtain the desired result.

\section{NECESSITY OF STRONG ANTICOMMUTATIVITY}

In this section, we show that the strong anticommutativity of the supercharges is necessary for a symmetric representation of the SUSY algebra (1.1) to be integrable. A basic fact is the following:

Theorem 3.1: Let $A$ and $B$ be self-adjoint operators in a Hilbert space $7{ }^{2}$ and $\mathscr{H}$ be a dense subspace of $\mathscr{H}$ left invariant by $A$ and $B$. Suppose that. $\mathscr{H}$ is a common core for $A^{2}$ and $B^{2}$, $A B+B A=0$ on $\mathscr{H}$, and $A^{2}$ and $B^{2}$ strongly commute. Then $A$ and $B$ strongly anticommute.

The idea of the proof of this theorem is to apply the following lemma:

Lemma 3.2: (Ref. 19) Let $A$ and $B$ be self-adjoint operators in a Hilbert space and $A=U_{A}|A|$, $B=U_{B}|B|$ be the polar decompositions of $A$ and $B$, respectively. Then $A$ and $B$ strongly anticommute if and only if (i) $U_{A} U_{B}=-U_{B} U_{A}$; (ii) $U_{A}|B| \subset|B| U_{A}$; (iii) $U_{B}|A| \subset|A| U_{B}$; (iv) $|A|$ and $|B|$ strongly commute.

The following lemma, which can be easily proven, is well known.

Lemma 3.3: Let $A$ and $B$ be self-adjoint operators in a Hilbert space. Suppose that $A$ is bounded. Then $A$ and $B$ strongly commute if and only if $A B \subset B A$.

Proof of Theorem 3.1: It is shown ${ }^{20}$ that 


$$
U_{A} f=\lim _{\varepsilon \downarrow 0} A\left(A^{2}+\varepsilon\right)^{-1 / 2} f, \quad f \in \mathscr{H} .
$$

The strong commutativity of $A^{2}$ and $B^{2}$ implies that of $\left(A^{2}+\varepsilon\right)^{-1 / 2}$ and $B^{2}$ for all $\varepsilon>0$. Hence, by Lemma 3.3, we have

$$
\left(A^{2}+\varepsilon\right)^{-1 / 2} B^{2} \subset B^{2}\left(A^{2}+\varepsilon\right)^{-1 / 2}
$$

for all $\varepsilon>0$. Note that

$$
B^{2} A f=A B^{2} f, \quad f \in \mathscr{A B} .
$$

Using these facts, we have for all $f, g \in \mathscr{L} \mathscr{C}$

$$
\begin{aligned}
\left(f, U_{A} B^{2} g\right) & =\lim _{\varepsilon \downarrow 0}\left(f, A B^{2}\left(A^{2}+\varepsilon\right)^{-1 / 2} g\right)=\lim _{\varepsilon \downarrow 0}\left(B^{2} A f,\left(A^{2}+\varepsilon\right)^{-1 / 2} g\right)=\lim _{\varepsilon \downarrow 0}\left(A B^{2} f,\left(A^{2}+\varepsilon\right)^{-1 / 2} g\right) \\
& =\lim _{\varepsilon \downarrow 0}\left(B^{2} f, A\left(A^{2}+\varepsilon\right)^{-1 / 2} g\right)=\left(B^{2} f, U_{A} g\right) .
\end{aligned}
$$

Since. $\mathscr{Z}$ is a core for $B^{2}$, it follows that $U_{A} g \in D\left(B^{2}\right)$ and $B^{2} U_{A} g=U_{A} B^{2} g$. By the condition that. $/ /$ is a core for $B^{2}$ again, this equality extends to all $g \in D\left(B^{2}\right)$, showing that $U_{A} B^{2} \subset B^{2} U_{A}$, which implies that $U_{A}$ and $B^{2}$ strongly commute (Lemma 3.3). In particular, we have

$$
\left(B^{2}+\varepsilon\right)^{-1 / 2} U_{A}=U_{A}\left(B^{2}+\varepsilon\right)^{-1 / 2} .
$$

Since $|B|=\sqrt{B^{2}}$, it follows that $U_{A}$ and $|B|$ strongly commute. Hence $U_{A}|B| \subset|B| U_{A}$. Similarly we can show that $U_{B}|A| \subset|A| U_{B}$. Moreover, we have for all $f, g \in \mathscr{A l}$

$$
\begin{aligned}
& \left(U_{A} f, U_{B} g\right)=\lim _{\varepsilon \mid 0}\left(\left(A^{2}+\varepsilon\right)^{-1 / 2} A f, U_{B} g\right) \\
& =\lim _{\varepsilon \downarrow 0}\left(A f, U_{B}\left(A^{2}+\varepsilon\right)^{-1 / 2} g\right) \\
& =\lim _{\varepsilon \downarrow 0} \lim _{\delta \downarrow 0}\left(\left(B^{2}+\delta\right)^{-1 / 2} B A f,\left(A^{2}+\varepsilon\right)^{-1 / 2} g\right) \\
& =\lim _{\varepsilon \downarrow 0} \lim _{\delta \downarrow 0}\left(B A f,\left(B^{2}+\delta\right)^{-1 / 2}\left(A^{2}+\varepsilon\right)^{-1 / 2} g\right) \\
& =-\lim _{\varepsilon \downarrow 0} \lim _{\delta \downarrow 0}\left(A B f,\left(B^{2}+\delta\right)^{-1 / 2}\left(A^{2}+\varepsilon\right)^{-1 / 2} g\right) \\
& =-\lim _{\varepsilon \downarrow 0} \lim _{\delta \downarrow 0}\left(B\left(B^{2}+\delta\right)^{-1 / 2} f, A\left(A^{2}+\varepsilon\right)^{-1 / 2} g\right)=-\left(U_{B} f, U_{A} g\right),
\end{aligned}
$$

which implies that $U_{A} U_{B}+U_{B} U_{A}=0$. Of course, $|A|$ and $|B|$ strongly commute. Thus we can apply Lemma 3.2 to obtain the desired result.

The main result in this section is the following:

Theorem 3.4: Let $\left\{\mathscr{H}, \mathcal{Z}, H, P, Q_{1}, Q_{2}\right\}$ be a symmetric representation of Eq. (1.1) and let $\hat{H}$ (resp. $\hat{P})$ be any self-adjoint extension of $H$ (resp. $P$ ). Suppose that, for $j=1,2, Q_{j}^{2}$ are essentially self-adjoint on $\mathscr{Z}$ and $\hat{H}$ and $\hat{P}$ strongly commute. Then each $Q_{j}$ is essentially self-adjoint on $\mathscr{D}$ and $\bar{Q}_{1}$ and $\bar{Q}_{2}$ strongly anticommute.

To prove this theorem, we need a lemma.

Lemma 3.5: Let $A$ be a symmetric operator in a Hilbert space and $\mathscr{Q}$ be a dense subspace of . . Suppose that $\mathscr{Q} \subset D\left(A^{2}\right)$ and $A^{2}$ is essentially self-adjoint on $\mathscr{L}$. Then $A$ is essentially selfadjoint on $\mathscr{L}$ and the operator equality 


$$
\overline{A^{2}}=\bar{A}^{2}
$$

holds.

Proof: This lemma is well known (e.g., Ref. 21, Chap. X, p. 341, Problem 28), but, to be self contained, we give a proof of it. Let $T=\overline{A^{2}}$. For all $f \in \mathscr{D}$, we have $\|A f\| \leqslant\|f\|\|T f\|$. Since $\mathscr{D}$ is a core for $T$, this inequality implies that $D(T) \subset D(\bar{A})$, where $\bar{A}$ is the closure of $A$ restricted to $\mathscr{Q}$, and

$$
\|\bar{A} f\| \leqslant\|f\|\|T f\|, \quad f \in D(T)
$$

It is obvious that

$$
(\bar{A} f, T g)=(T f, \bar{A} g), \quad f, g \in \mathscr{D} .
$$

By Eq. (3.2) and the condition that $\mathscr{D}$ is a core for $T$, we can show that Eq. (3.3) extends to all $f, g \in D(T)$. Thus we can apply Lemma 2.5 to conclude that $\bar{A}$ is self-adjoint. We have $A^{2} \subset \bar{A}^{2}$. Hence $T \subset \bar{A}^{2}$. Since the both sides are self-adjoint, Eq. (3.1) follows.

Proof of Theorem 3.4: We have from Eq. (1.1)

$$
Q_{1}^{2} \uparrow \mathscr{D C} \hat{H}+\hat{P}, \quad Q_{2}^{2}\lceil\mathscr{Q} \subset \hat{H}-\hat{P} .
$$

By Lemma $3.5, Q_{j}$ is essentially self-adjoint on $\mathscr{Q}$ and

$$
\overline{Q_{j}^{2}}=\bar{Q}_{j}^{2}, \quad j=1,2
$$

Hence we obtain

$$
\bar{Q}_{1}^{2}=\overline{\hat{H}+\hat{P}}, \quad \bar{Q}_{2}^{2}=\overline{\hat{H}-\hat{P}} .
$$

Since $\{\hat{H}, \hat{P}\}$ is a family of strongly commuting self-adjoint operators, Eq. (3.4) implies that $\bar{Q}_{1}^{2}$ strongly commutes with $\bar{Q}_{2}^{2}$. Hence, by Theorem $3.1, \bar{Q}_{1}$ and $\bar{Q}_{2}$ strongly anticommute.

Theorem 3.4 implies the following: Let $\left\{\not{F}, \mathscr{Q}_{,}, P, Q_{1}, Q_{2}\right\}$ be a symmetric representation of Eq. (1.1). Suppose that, for $j=1,2, Q_{j}^{2}$ are essentially self-adjoint on $\mathscr{D}$ (then, by Lemma $3.5, Q_{j}$ is essentially self-adjoint on $\mathscr{D})$, but $\widehat{Q}_{1}$ and $\bar{Q}_{2}$ do not strongly anticommute. Then $\{\hat{H}, \hat{P}\}$ cannot be a family of strongly commuting self-adjoint operators (in particular, $\{\hat{H}, \hat{P}\}$ does not satisfy the spectral condition).

In Theorem 3.4 , the condition that $Q_{j}^{2}(j=1,2)$ is essentially self-adjoint on $\mathscr{Q}$ is not so restrictive for applications. On the other hand, it is natural to require, as a condition of (weaker) integrability of a symmetric representation $\left\{\mathscr{H}, \mathscr{D}, H, P, Q_{1}, Q_{2}\right\}$ of Eq. (1.1), that there exist selfadjoint extensions $\hat{H}$ and $\hat{P}$ of $H$ and $P$, respectively, such that $\{\hat{H}, \hat{P}\}$ is a family of strongly commuting self-adjoint operators (otherwise the concept of the spectral condition could not be defined properly). In this sense, Theorem 3.4 tells us that the strong anticommutativity of the supercharges is necessary for integrability of the SUSY algebra $\left\{\mathscr{H}, \mathscr{Z}, H, P, Q_{1}, Q_{2}\right\}$.

We conclude this section with a counterexample of a symmetric representation of the SUSY algebra (1.1) in which each $Q_{j}^{2}$ is essentially self-adjoint on $\mathscr{D}$, but, $\bar{Q}_{1}$ and $\bar{Q}_{2}$ do not strongly anticommute. Although the example given below is taken from supersymmetric quantum mechanics, not from SSQFT, it is interesting both mathematically and physically. ${ }^{22,23}$

Example: Let $\left\{\mathbf{a}_{\nu}\right\}_{\nu=1}^{n}$ be a finite set of isolated points in $\mathbf{R}^{2}$ and set $M:=\mathbf{R}^{2}\left\{\left\{\mathbf{a}_{\nu}\right\}_{\nu=1}^{n}\right.$. Let

$$
A_{1}(\mathbf{r})=-\sum_{\nu=1}^{n} \sum_{\alpha, \beta=0}^{m} C_{\alpha, \beta}^{(\nu)} D_{x}^{\alpha} D_{y}^{\beta}\left(\frac{y-a_{\nu 2}}{\left|\mathbf{r}-\mathbf{a}_{\nu}\right|^{2}}\right)
$$




$$
A_{2}(\mathbf{r})=\sum_{\nu=1}^{n} \sum_{\alpha, \beta=0}^{m} C_{\alpha, \beta}^{(\nu)} D_{x}^{\alpha} D_{y}^{\beta}\left(\frac{x-a_{\nu 1}}{\left|\mathbf{r}-\mathbf{a}_{\nu}\right|^{2}}\right), \quad \mathbf{r}=(x, y) \in M
$$

where $C_{\alpha, \beta}^{(\nu)}$ are real constants, $m \geqslant 0$ is an integer, and $D_{x}$ and $D_{y}$ are the distributional partial differential operators in $x$ and $y$, respectively. Note that

$$
D_{x} A_{2}(\mathbf{r})-D_{y} A_{1}(\mathbf{r})=2 \pi \sum_{\nu=1}^{n} \sum_{\alpha, \beta=0}^{m} C_{\alpha, \beta}^{(\nu)} D_{x}^{\alpha} D_{y}^{\beta} \delta\left(\mathbf{r}-\mathbf{a}_{\nu}\right),
$$

where $\delta(\mathbf{r})$ is the Dirac delta distribution on $\mathbf{R}^{2}$. Let

$$
P_{1}=-i D_{x}-A_{1}, \quad P_{2}=-i D_{y}-A_{2}
$$

acting in $L^{2}\left(\mathbf{R}^{2}\right)$. Obviously each $P_{j}$ leaves $C_{0}^{\infty}(M)$ invariant. It follows from Eq. (3.5) that

$$
P_{1} P_{2} f=P_{2} P_{1} f, \quad f \in C_{0}^{\infty}(M) .
$$

In Ref. 22 we proved that $P_{j}$ is essentially self-adjoint on $C_{0}^{\infty}(M)$.

Let $\sigma_{j}, j=1,2$, be the first two of the Pauli matrices

$$
\sigma_{1}=\left(\begin{array}{cc}
0 & 1 \\
1 & 0
\end{array}\right), \quad \sigma_{2}=\left(\begin{array}{cc}
0 & -i \\
i & 0
\end{array}\right)
$$

In the Hilbert space

$$
\mathscr{H}:=L^{2}\left(\mathbf{R}^{2}\right) \otimes \mathbf{C}^{2}=\left\{\left(\begin{array}{l}
f \\
g
\end{array}\right) \mid f, g \in L^{2}\left(\mathbf{R}^{2}\right)\right\}
$$

we define the self-adjoint operators

$$
Q_{j}=\sigma_{j} \otimes \bar{P}_{j}, \quad j=1,2 .
$$

Let

$$
\mathscr{D}=\left\{\left(\begin{array}{l}
f \\
g
\end{array}\right) \mid f, g \in C_{0}^{\infty}(M)\right\} .
$$

Then $\mathscr{Z}$ is dense in $\mathscr{H}$ and each $Q_{j}$ is essentially self-adjoint on $\mathscr{D}$. Moreover, $Q_{j}$ leaves $\mathscr{D}$ invariant. By Eq. (3.6) and the anticommutativity of $\sigma_{1}$ and $\sigma_{2}$, we have

$$
Q_{1} Q_{2} \psi+Q_{2} Q_{1} \psi=0, \quad \psi \in \mathscr{D}
$$

Let

$$
H:=\frac{1}{2}\left(I \otimes \bar{P}_{1}^{2}+I \otimes \bar{P}_{2}^{2}\right), \quad P:=\frac{1}{2}\left(I \otimes \bar{P}_{1}^{2}-I \otimes \bar{P}_{2}^{2}\right)
$$

Then we can easily see that the first two equations of (1.1) hold on $\mathscr{T}$. It follows that $\left\{. \mathcal{F}, Q_{,}, P, P, Q_{1}, Q_{2}\right\}$ is a symmetric representation of the SUSY algebra (1.1).

In the same way as in Ref. 22 , we can show that each $Q_{j}^{2}$ is essentially self-adjoint on $\mathscr{Z}$. By Theorem 3.4 in Ref. $10, Q_{1}$ and $Q_{2}$ strongly anticommute if and only if $\bar{P}_{1}$ and $\bar{P}_{2}$ strongly commute. On the other hand, it was shown in Ref. 22 that $\bar{P}_{1}$ and $\vec{P}_{2}$ strongly commute if and only if the constant $C_{0,0}^{(\nu)}$ is an integer for all $\nu=1, \ldots, n$ (in this case we say that the magnetic flux is locally quantized). Hence, if the magnetic flux is not locally quantized, then $Q_{1}$ and $Q_{2}$ do not 
strongly anticommute. Thus, by Theorem 3.4, any self-adjoint extension of $\{H, P\}$ is not a family of strongly commuting self-adjoint operators if the magnetic flux is not locally quantized. It also follows that, under the same condition, $H$ is not essentially self-adjoint on $\mathscr{P}$ (by Theorem 2.2).

Remark: The operator $Q:=Q_{1}+Q_{2}$ is called a Dirac-Weyl operator. In the case where the magnetic flux is not locally quantized, it has nontrivial and interesting properties, see Ref. 23 for the details.

\section{REPRESENTATION BASED ON TWO STRONGLY ANTICOMMUTING SELF-ADJOINT OPERATORS}

In this section, we shall show that the strong anticommutativity of the self-adjoint supercharges is sufficient to construct an integrable symmetric representation of the SUSY algebra (1.1).

Let $Q_{1}$ and $Q_{2}$ be strongly anticommuting self-adjoint operators in a Hilbert space $\mathscr{H}$ and let $E_{1}$ and $E_{2}$ be the spectral measures of $\left|Q_{1}\right|$ and $\left|Q_{2}\right|$, respectively. Then, by Lemma 2.3(iii), we can define a two-dimensional spectral measure by

$$
E=E_{1} \otimes E_{2}
$$

By Lemma 2.3(i) (or the functional calculus)

$$
H:=\frac{1}{2}\left(Q_{1}^{2}+Q_{2}^{2}\right)=\frac{1}{2} \int_{\mathbf{R}^{2}}\left(\lambda^{2}+\mu^{2}\right) d E(\lambda, \mu)
$$

is a non-negative self-adjoint operator. It follows from Lemma 2.3(iii) and the functional calculus that $Q_{1}^{2}-Q_{2}^{2}\left(=\left|Q_{1}\right|^{2}-\left|Q_{2}\right|^{2}\right)$ is essentially self-adjoint. Hence we can define the self-adjoint operator

$$
P:=\frac{1}{2} \overline{\left(Q_{1}^{2}-Q_{2}^{2}\right)}=\int_{\mathbf{R}^{2}}\left(\lambda^{2}-\mu^{2}\right) d E(\lambda, \mu)
$$

Let

$$
\mathscr{D}=\bigcup_{0 \leqslant a<b<\infty, 0 \leqslant c<d<\infty} \operatorname{Ran} E([a, b] \times[c, d]),
$$

where $\operatorname{Ran} T$ denotes the range of the operator $T$. Then $\mathscr{Q}$ is a dense subspace of $\mathscr{H}$ and left invariant by $\left|Q_{j}\right|, j=1,2$.

Lemma 4.1: The set $\left\{\not Z, Q D, H, P, Q_{1}, Q_{2}\right\}$ is a symmetric representation of $E q .(1.1)$.

Proof: Let $Q_{j}=U_{j}\left|Q_{j}\right|(j=1,2)$ be the polar decomposition of $Q_{j}$. Then, by Lemma 3.2, $U_{j}$ commutes with $E_{k}(j, k=1,2)$. Hence it follows that $H, P, Q_{1}$, and $Q_{2}$ leaves $\mathscr{D}$ invariant, satisfying the first two equations in (1.1) on $\mathscr{P}$. The third equation in (1.1) follows from condition (i) in Lemma 3.2.

Lemma 4.2: The subspace $\mathscr{D}$ is a core for $H$.

Proof: Let $\psi \in D(H)$ and $\psi_{n}=E([0, n] \times[0, n]) \psi, n=1,2, \ldots$ Then $\psi_{n} \in \mathscr{D}$. By the functional calculus, one can easily show that $\psi_{n} \rightarrow \psi, H \psi_{n} \rightarrow H \psi$ as $n \rightarrow \infty$. Thus the desired result follows.

By Lemmas 4.1 and 4.2 , we can apply Theorem 2.2 to obtain the following result:

Theorem 4.3: Let $\left\{\mathscr{H}, \mathscr{C}, H, P, Q_{1}, Q_{2}\right\}$ be as above. Then the conclusion of Theorem 2.2 holds with $\bar{H}, \bar{P}, \bar{Q}_{1}$, and $\bar{Q}_{2}$ replaced by $H, P, Q_{1}$, and $Q_{2}$, respectively. 


\section{ACKNOWLEDGMENT}

This work is supported by a Grant-In-Aid No. 06640188 for science research from the Ministry of Education, Japan.

'P. G. O. Freund, Introduction to Supersymmetry (Cambridge University, Cambridge, England, 1986).

${ }^{2} \mathrm{~A}$. Jaffe and A. Lesniewski, "Supersymmetric quantum fields and infinite-dimensional analysis," in Nonperturbative Quantum Field Theory, edited by G.'t Hoot, A. Jaffe, G. Mack, P. K. Mitter, and R. Stora (Plenum, New York, 1988).

${ }^{3}$ A. Jaffe, A. Lesniewski, and C. Wieczerkowski, "Supersymmetry and the spectral condition on a cylinder," Lett. Math. Phys. 16, 385-388 (1988).

${ }^{4}$ A. Arai, "A general class of infinite-dimensional Dirac operators and path inlegral representation of their index," $\mathrm{J}$. Funct. Anal. 105, 342-408 (1992).

${ }^{5}$ A. Arai, "Dirac operators in Boson-Fermion Fock spaces and supersymmetric quantum field theory," J. Geom. Phys. 11, 465-490 (1993).

${ }^{6}$ F.-H. Vasilescu, “Anticommuting self-adjoint operators,” Rev. Roum. Math. Pures Appl. 28, 77-91 (1983).

'Yu. S. Samoilenko, Spectral Theory of Families of Self-Adjoint Operators (Kluwer Academic, Dordrecht, 1991).

${ }^{8}$ S. Pedersen, "Anticommuting self-adjoint operators," J. Funct. Anal. 89, 428-443 (1990).

${ }^{9}$ A. Arai, "Commutation properties of anticommuting self-adjoint operators, spin representation, and Dirac operators," Integr. Eq. Oper. Th. 16, 38-63 (1993).

${ }^{10}$ A. Arai, "Characterization of anticommutativity of self-adjoint operators in connection with Clifford algebra and applications," Integr. Eq. Oper. Th. 17, 451-563 (1993).

"A. Arai, "Analysis on anticommuting self-adjoint operators," Adv. Stud. Pure Math. 23, 1-15 (1994).

${ }^{12}$ The situation is similar to the case of commutativity for unbounded self-adjoint opcrators (sce, e.g., Sec. VIII.5 in Ref, 17).

${ }^{13}$ See also Ref. 16.

${ }^{14}$ See, e.g., Ref. 1.

${ }^{15}$ See Ref, 2 and references therein.

${ }^{16}$ A. Arai, "Fock space representations of the relativistic supersymmetry algebra in the two-dimensional space-time," Hokkaido University Preprint Series in Math. No. 123, 1991.

${ }^{17}$ M. Reed and B. Simon, Functional Analysis, Methods of Modern Mathematical Physics Vol. I (Academic, New York, 1972).

${ }^{18}$ A. E. Nussbaum, "A commutativity theorem for unbounded operators in Hilbert space," Trans. Am. Math. Soc. 140, 485-491 (1969). See also Proposition 4.1 in Ref. 8.

${ }^{19}$ Corollary 3.5 in Ref. 8.

${ }^{20}$ Lemma 3.1 in Ref. 9.

${ }^{21}$ M. Reed and B. Simon, Fourier Analysis, Self-Adjointness, Methods of Modern Mathematical Physics Vol. II (Academic, New York, 1975).

${ }^{22}$ A. Arai. "Momentum operators with gauge potentials, local quantization of magnetic flux, and representation of canonical commutation relations," J. Math. Phys. 33, 3374-3378 (1992).

${ }^{23}$ A. Arai, "Properties of the Dirac-Weyl operator with a strongly singular gauge potential," J. Math. Phys. 34, 915-935 (1993). 